Original Research Paper

\title{
Blind Pre-Coder for MIMO System Capacity Enhancement
}

\author{
${ }^{1}$ Eman Al Khader and ${ }^{2}$ Shereen S. Ismail \\ ${ }^{I}$ Department of Electrical Engineering, University of Jordan, Jordan \\ ${ }^{2}$ Department of Computer Science and Engineering, American University of Ras Al-Khaimah, UAE
}

\author{
Article history \\ Received: 17-10-2016 \\ Revised: 02-12-2016 \\ Accepted: 11-01-2017 \\ Corresponding Author: \\ Shereen S. Ismail \\ Department of Computer \\ Science and Engineering, \\ American University of Ras \\ Al-Khaimah, UAE \\ Mob: +971505373477 \\ Fax: +97172210638 \\ Email: support@thescipub.com
}

\begin{abstract}
In this study, linear scheme at the transmitter is proposed as a blind pre-coder to improve the performance and enhance the channel capacity of MIMO systems with certain configurations for transmitting and receiving antennas over fading channels. This is accomplished by generating a specific linear pre-coder matrix without having any prior information about the Channel State (CS) matrix. Simulation results have demonstrated the effectiveness of this linear pre-coder at the transmitter to enhance the MIMO channel capacity for all types of correlations.
\end{abstract}

Keywords: MIMO, Channel Capacity, Channel State, Pre-Coder, Blind

\section{Introduction}

In response to fast growing demands for wireless capacity in mobile communications, driven by cellular mobile networks and broadband wireless internet service providers with rich multimedia content worldwide and due to the limited available radio spectrum, the communication capacity demands cannot be targeted without introducing new technologies for efficient use of communication spectral. Advances in coding, made it possible to approach the Shannon capacity upper limit in systems equipped with a single antenna link. Further significant advances in spectral efficiency are available without adding more bandwidth or increasing transmitted power over the antenna through the use of which is known as a MIMO system (Zheng et al., 2015; Rusek et al., 2013) where multiple antennas at both sides (i.e., transmitter $T_{x}$ and receiver $R_{x}$ ) have been employed.

A MIMO is a smart form of multi-element array antennas in which the MIMO channel propagation scenario is modeled as individual channels between any given pairs of $T_{x}$ and $R_{x}$ antennas which are modeled by an independent flat fading process as it is common in communication environments in the absence of scattering where a signal transmitted from every individual $T_{x}$ antenna appears uncorrelated at each of the $R_{x}$ antennas.

In addition, the combination of MIMO and OFDM technologies became a powerful key in recent broadband wireless communication in order to achieving higher data rate and larger system capacity over mobile wireless links. In (Zaier and Bouallègue, 2012), the authors have presented an enhancement of the blind channel estimation in a MIMO-OFDM context operating over frequency selective fading channels. The enhanced estimator performance was investigated by computer simulations and proved its effectiveness when compared to other channel estimation schemes.

The pre-coder design for the pre-coding using blind channel estimation for MIMO-OFDM systems have been considered in (Noh et al., 2014). The authors have presented a new pre-coder built upon on a sparse structure. The positive point of the proposed pre-coder is that it is useful for enhancing data rate with MIMO precoding especially when minimal subcarriers is used in order to accomplish signal correlation that is necessary for blind channel estimation without affecting most of the subcarriers. The proposed pre-coder design uses partial channel state information at $T_{x}$ through limited requirement for feedback.

The use of a full rank pre-coder matrix was considered in (Cho et al., 2010) for channel estimation in subspace while in (Jung, 2012) a multi-mode pre-coding scheme was presented through minimizing the interference in which the proposed pre-coder maximize the total system capacity with mode selection.

In this study, we present a linear scheme at the $T_{x}$ to enhance the performance in MIMO systems under specific configurations at $T_{x}$ and $R_{x}$ antennas over fading channels. We generate a specific linear precoder matrix after applying a condensed searching algorithm without having any prior information about CS matrix. After conducting many simulation 
experiments, the results eventually showed that the use of the proposed blind pre-coder can maximize the MIMO system capacity for certain limit.

The rest of this paper is organized as follows. Section II the proposed blind pre-coder system is presented in details. Section III, we discuss and analyze the effectiveness of the proposed system by conducting many MATLAB simulation experiments. We conclude our paper by section IV.

\section{System Description}

With $n_{T}$ at $T_{x}$ and $n_{R}$ at $R_{x}$ antennas, we can write the attenuation that the signal faces in its way from the $T_{x}$ to the $R_{x}$ by $H$ matrix as follows:

$$
H=\left(\begin{array}{cccc}
h_{1,1} & h_{1,2} & \cdots & h_{1, n_{T}} \\
h_{2,1} & h_{2,2} & \cdots & \vdots \\
\cdots & \cdots & \cdots & \vdots \\
h_{n_{R}, 1} & \cdots & \cdots & h_{n_{R}, n_{T}}
\end{array}\right)
$$

where, $H$ is the $n_{R} \times n_{T}$ channel matrix with components modelled as independent and identically-distributed (iid) Zero Mean unit variance generally known as Circularly Symmetric Complex Gaussian random variables (ZMCSCG) where $h_{i, j}$ is the fading coefficient from the $j$ th at $T_{x}$ antenna to the $i$ th at $R_{x}$ antenna. Generally, MIMO system is presented in matrix form as:

$$
y=H x+n
$$

where, $y$ is the $n_{R} \times 1$ vector of signals received on the $n_{R}$ antennas, $x$ is the $n_{T} \times 1$ vector of signals transmitted on the $n_{T}$ at $T_{x}$ antennas, $n$ is the $n_{R} \times 1$ noise vector consisting of independent complex Gaussian distributed elements with zero mean and variance $\sigma^{2}$. We assume a channel bandwidth of $B$ where $\sigma^{2}=N_{0} B$. For simplicity given a transmit power constrain of $E_{s}$ we will use an equivalent model with a unity noise power and transmit power of $\frac{E_{s}}{\sigma_{n}^{2}}=\rho$ where $\rho$ is the average SNR per $R_{x}$ antenna under unity channel gain.

The capacity for this system is shown to be:

$$
C=E\left\{\log _{2} \operatorname{det}\left(I_{m}+\frac{1}{\sigma^{2}} H R_{x x} H^{H}\right)\right\} b p s / H z
$$

where, $E$ denotes expectation, superscript $H^{H}$ represents transpose conjugate, $I_{m}$ represent $m \times m$ identity matrix where $m$ is the $\min \left(n_{R}, n_{T}\right)$. Assuming equal power distribution we can replace $R_{x x}$ which determines the covariance of the MIMO channel input; by $\frac{E_{s}}{n_{T}}$ and the final capacity Equation for equal power transmission is:

$$
C=E\left\{\log _{2} \operatorname{det}\left(I_{m}+\frac{\rho}{n_{T}} H H^{H}\right)\right\} b p s / H z
$$

In (Foschini and Gans, 1998) showed that the capacity $C$; in case of a fixed transmitter power and bandwidth; increases linearly with $\min \left(n_{R}, n_{T}\right)$. In other words, without increasing the transmit power or bandwidth the capacity of the wireless channel can be increased by simply increasing the number of $T_{x}$ and $R_{x}$ antennas.

Actually, Shannon (Wang et al., 2014) represents the maximum data rate to be transmitted over the communication channel with the minimum error probability.

The MIMO capacity relation is reached by maximizing the mutual information in all covariance matrices inputted and at the same time settling the power constrain, so:

$C=\max _{\operatorname{Tr}\left(R_{x x}\right)=E_{s}} B \log _{2}\left[I_{n_{R}}+H R_{x x} H^{H}\right]$

If we consider the Singular Value Decomposition (SVD) of the channel matrix $H$ as in (Shiu et al., 2000) and uniform power transmitted from all $n_{T}$ antennas are supposed, where it is a good choice for power distribution since it is independent on $H$ and by so it is not mandatory to know the channel at the $T_{x}$ side and that reduces system complexity and in case of equal power transmission from the $n_{T}$ at $T_{x}$ antennas, the channel capacity will be:

$$
C=E\left\{\log _{2} \operatorname{det}\left(I_{m+} \frac{E_{s}}{\sigma^{2} n_{T}} Q\right)\right\}
$$

where, $Q$ is the Wish art matrix defined as:

$$
Q=\left\{\begin{array}{c}
H H^{H} n_{R}<n_{T} \\
H^{H} H, n_{R} \geq n_{T}
\end{array}\right\}
$$

In order to go on designing a pre-coder for our system, we should investigate the effect of the pre-coder $(F)$ on the MIMO system capacity formula:

$\hat{y}=H F x+n$

$\mathrm{F}$ is the $n_{T} \times m$ pre-coder matrix, where $m=\operatorname{rank}(H)$ $\leq \min \left(n_{R}, n_{T}\right)$ is the number of parallel streams of data at $T_{x}$. Assuming a system of uncorrelated input sources, each normalized to unit power. The equivalent channel matrix can be expressed by:

$H_{e q}=H F$ 
The capacity of this system taking equal power transmission in consideration is:

$$
\begin{aligned}
& C=E\left\{\log _{2} \operatorname{det}\left(I_{m+} \frac{E_{s}}{\sigma^{2} n_{T}} Q\right)\right\} \\
& Q=\left\{\begin{array}{l}
H_{e q} H_{e q}^{H} n_{R}<n_{T} \\
H_{e q}^{H} H_{e q} n_{R} \geq n_{T}
\end{array}\right\} \\
& Q=\left\{\begin{array}{cc}
H F F^{H} H^{H} n_{R}<n_{T} \\
F^{H} H^{H} H F & n_{R} \geq n_{T}
\end{array}\right\} \text { where } \operatorname{tr}\left(F F^{H}\right) \leq E_{s}
\end{aligned}
$$

\section{Power Consideration}

The sent signal power $E_{s}$ is determined by trace $\left(R_{x x}\right)$ where $R_{x x}$ is the covariance matrix of the sent data. We use the assumption that the sent data to be iid Guassian random variables where $R_{x x}=\frac{E_{s}}{n_{T}} I_{n_{T}}$ and it had been assumed that the received signal power by each receiving antenna equals the total transmitted power $E_{s}$ and that can be assured by:

$$
\sum_{j=1}^{n_{T}}\left|h_{i, j}\right|^{2}=n_{T}, i=1,2, \ldots, n_{R}
$$

Furthermore, the power constraint $\left(\operatorname{trace}\left(F F^{F}\right) \leq E_{s}\right)$ should be imposed on the pre-coder $\mathrm{F}$ to limit the total transmitted power.

\section{Correlated Channel Model}

At the $R_{x}$ side the signal components may have correlation because of the limited distance between antenna elements or applying same scattering conditions. This correlation degrades the MIMO system capacity. The channel matrix due to the existence of this correlation is:

$$
\operatorname{vec}(H)=R^{1 / 2} \operatorname{vec}\left(H_{w}\right)
$$

where, $H_{w}$ is the channel matrix for the non-correlated channels, vec stands for vectorization and $R$ is the covariance matrix of the channel of dimension $n_{R} n_{T} \times$ $n_{R} n_{T}$, so:

$$
R=E\left\{\operatorname{vec}(H) \operatorname{vec}(H)^{H}\right\}
$$

The channel matrix can be expressed by:

$$
H=R_{a, R}^{1 / 2} H_{w} R_{a, T}^{1 / 2}
$$

where, $R_{R}$ is the reception correlation matrix and $R_{T}$ is the transmission correlation matrix, whereas expressed before $R_{a, T}$ and $R_{a, R}$ are of the form (Hjørungnes, 2005):

$$
R_{a, T}=\left(\begin{array}{cccc}
1 & \rho_{1,1} & \cdots & \rho_{1, n T} \\
\rho_{1,1} & 1 & \cdots & \vdots \\
\cdots & \cdots & \cdots & \vdots \\
\rho_{n T, 1} & \cdots & \cdots & 1
\end{array}\right)
$$

\section{Performance Evaluation}

This work is a continued research to the published work in (Al-Khader and Ismail, 2015), in which we were looking for a design that can achieve the highest capacity enhancement for MIMO systems were the mentioned one that used the partial channel knowledge was the best choice. In this study, we design a blind pre-coder that has no prior knowledge about the channel matrix and by so no need for a feedback link from the $R_{x}$ to the $T_{x}$.

As this research aims is to find a matrix that can be applied with correlated channels, we started with conducting a condensed searching algorithm in order to find the best matrices as a pre-coding step for a different types of correlated channels and with different amounts of correlation. Design also take into consideration the possibility of errors occurrences in estimating the correlation matrix so the pre-coder we are searching for is stable and will achieve stability even if the correlation in real environment is higher or less than the parameters values assumed in our experiments.

The design considers MIMO system of $3 \times 3$ dimension and the effect of adding a pre-coder matrix for different correlation cases and different correlation values ranging from $75-95 \%$ in general. The general flowchart that describe the search algorithm of the precoder matrix is shown in Fig. 1.

After taking a look on the generated F, matrices we found a pre-coder matrix that made a considerably good capacity enhancement for all cases of correlation, which has 0.7 Determinant, Rank of 3 condition number equals 0.276 and trace of 3 :

$F=\left[\begin{array}{ccc}j 0.4 & j 0.4 & -j 0.4 \\ -j 0.4 & j 0.4 & j 0.4 \\ 0.4 & -j 0.4 & 0.4\end{array}\right]$

Figure 2 to 5 are samples of the results found using the above proposed algorithm in compared with uncorrelated channel, correlated channel, correlated channel with precoder matrix and fully correlated channel.

We study the capacity enhancement for all types of column correlation and row correlation. In most cases the results shows that the range of correlation values are from $95-75 \%$. 


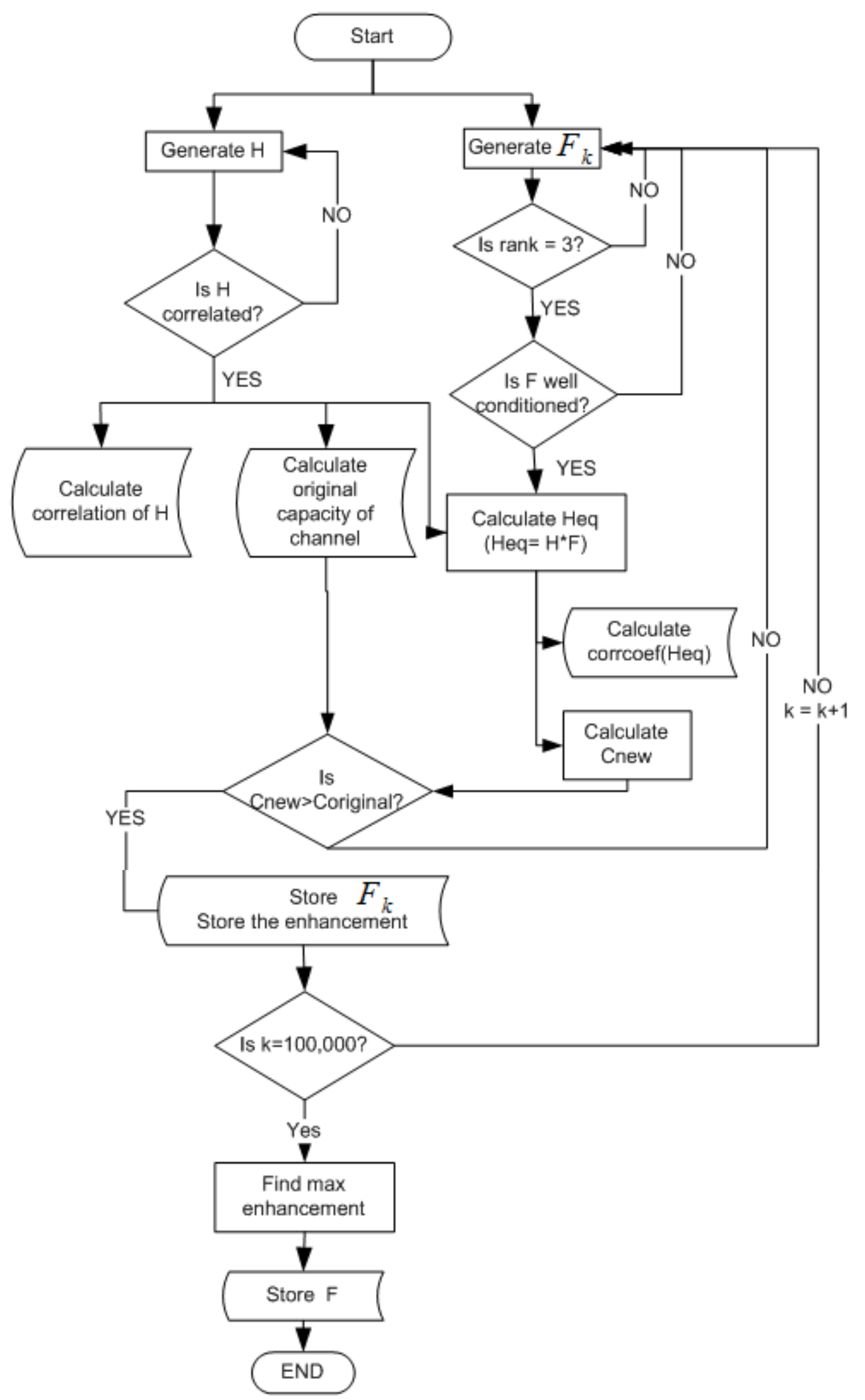

Fig. 1. Searching algorithmic for finding $F$ 


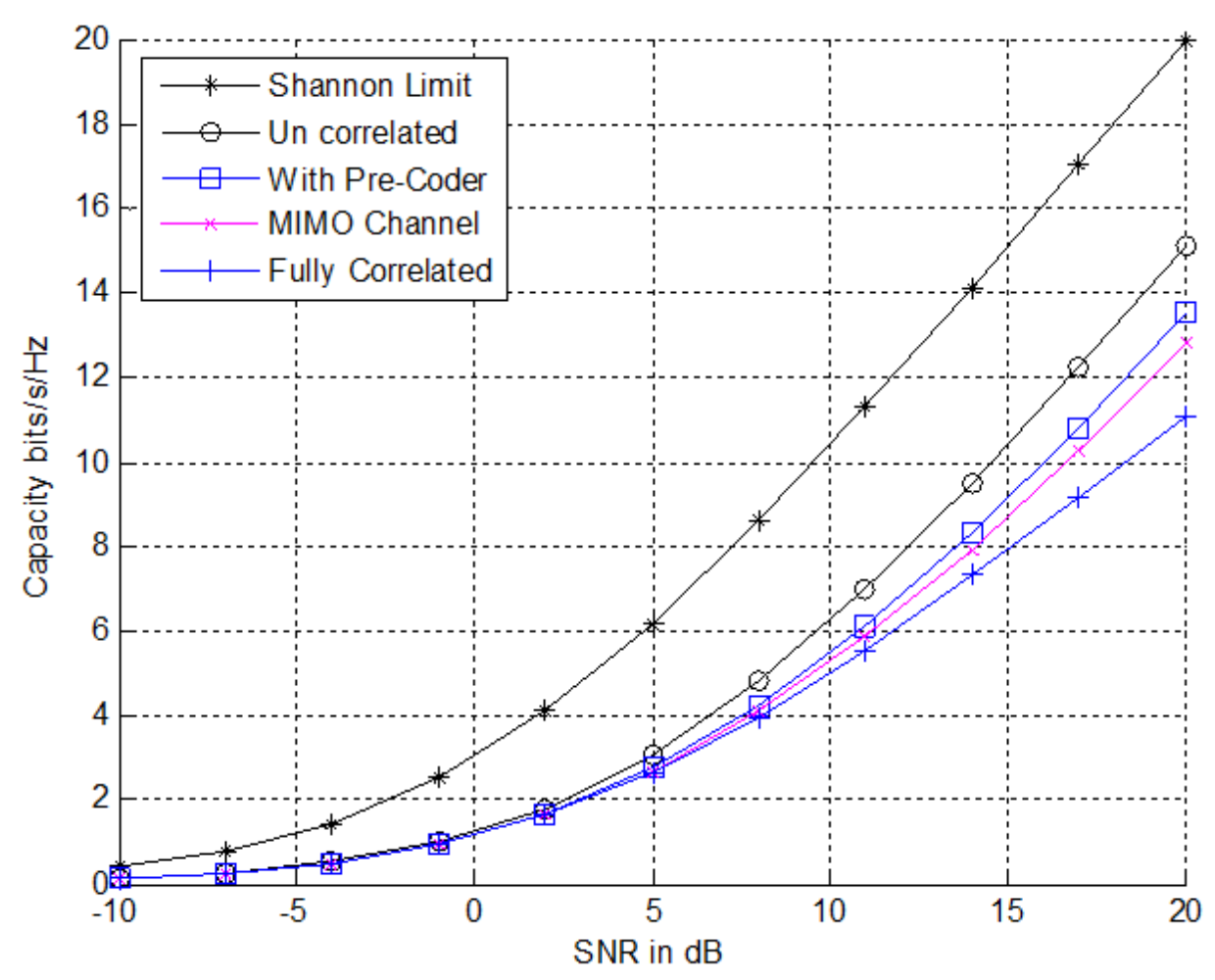

Fig. 2. MMO system capacity for two correlated columns

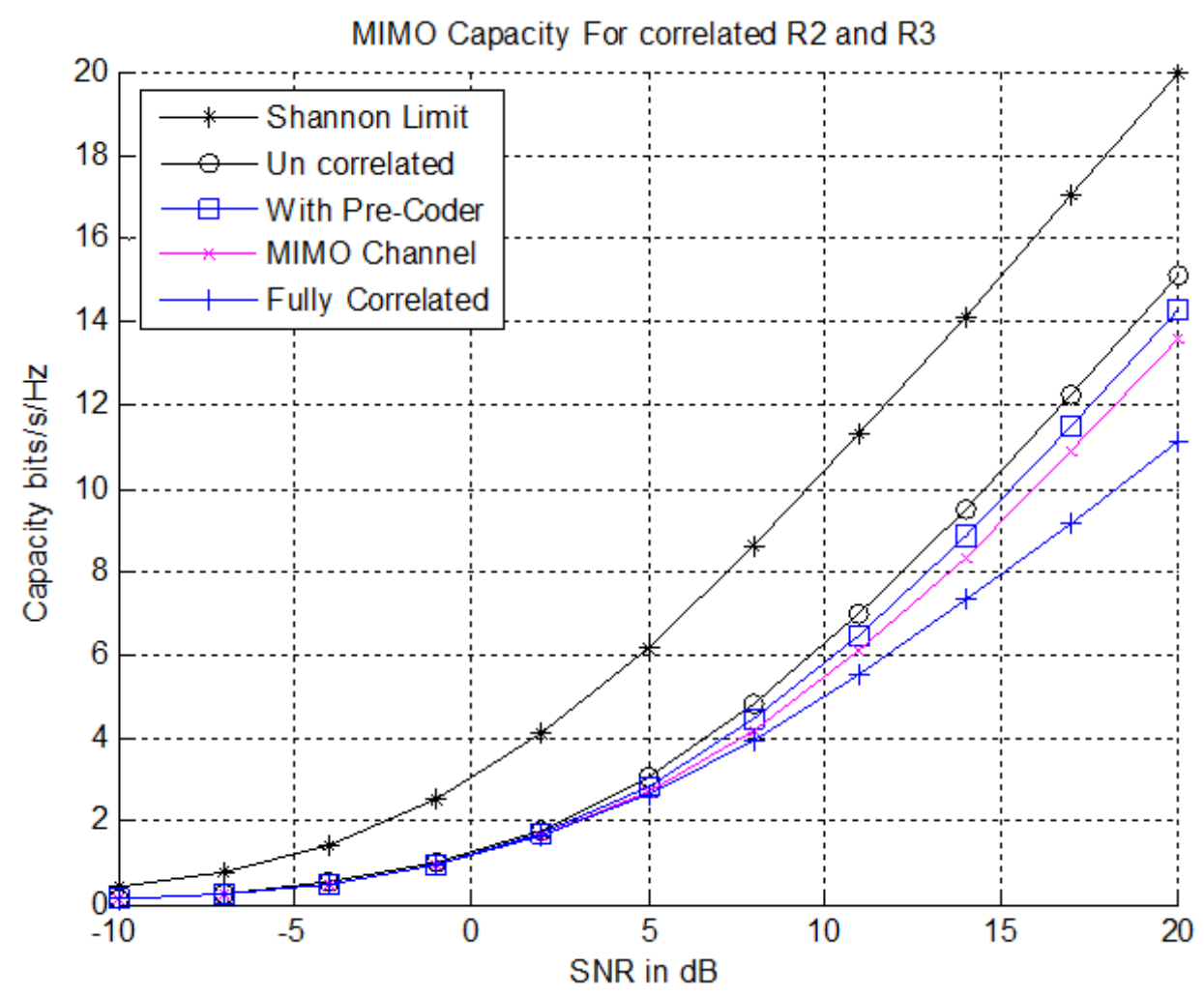

Fig. 3. MMO system capacity for correlated rows 


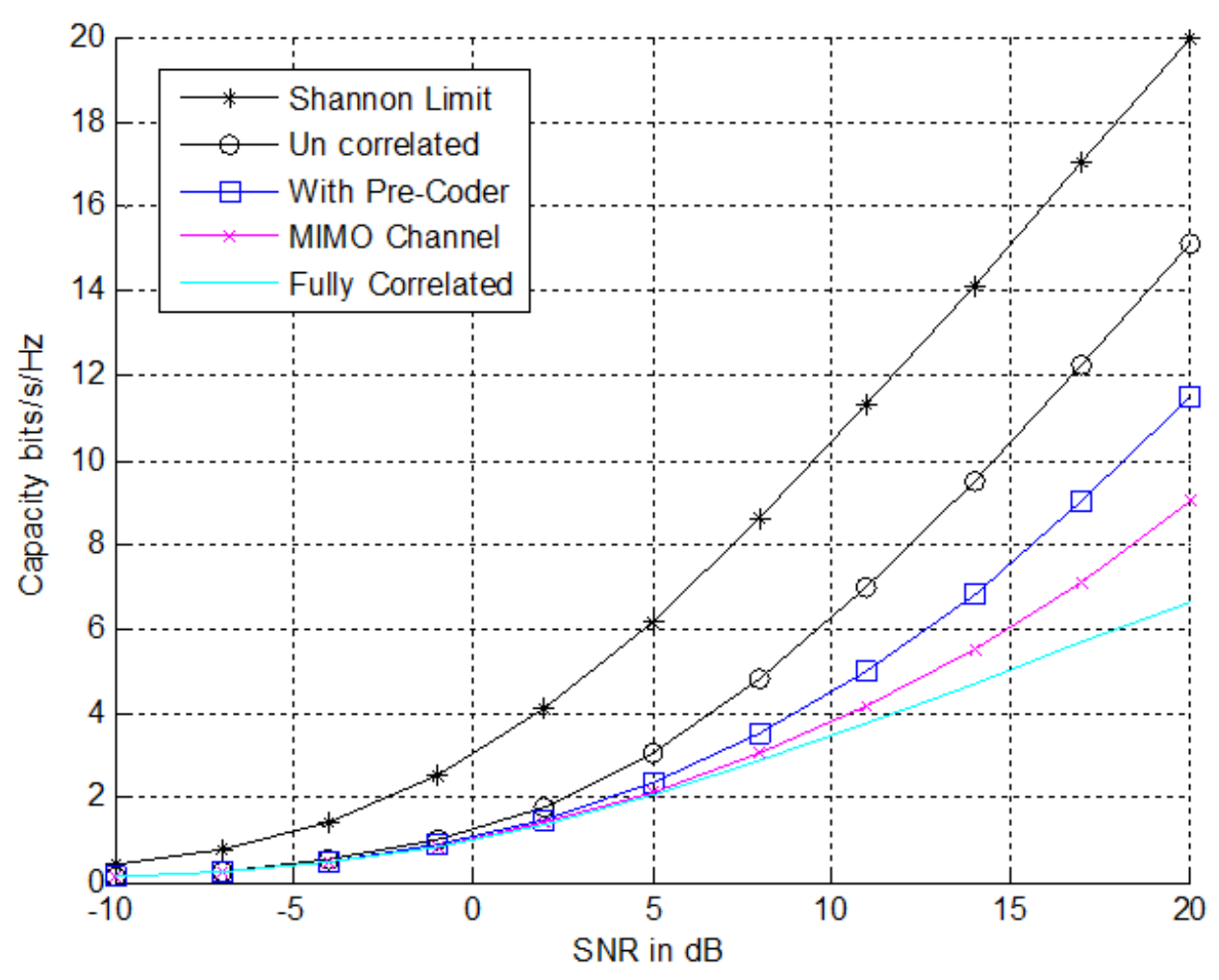

Fig. 4. MIMO capacity for three correlated columns

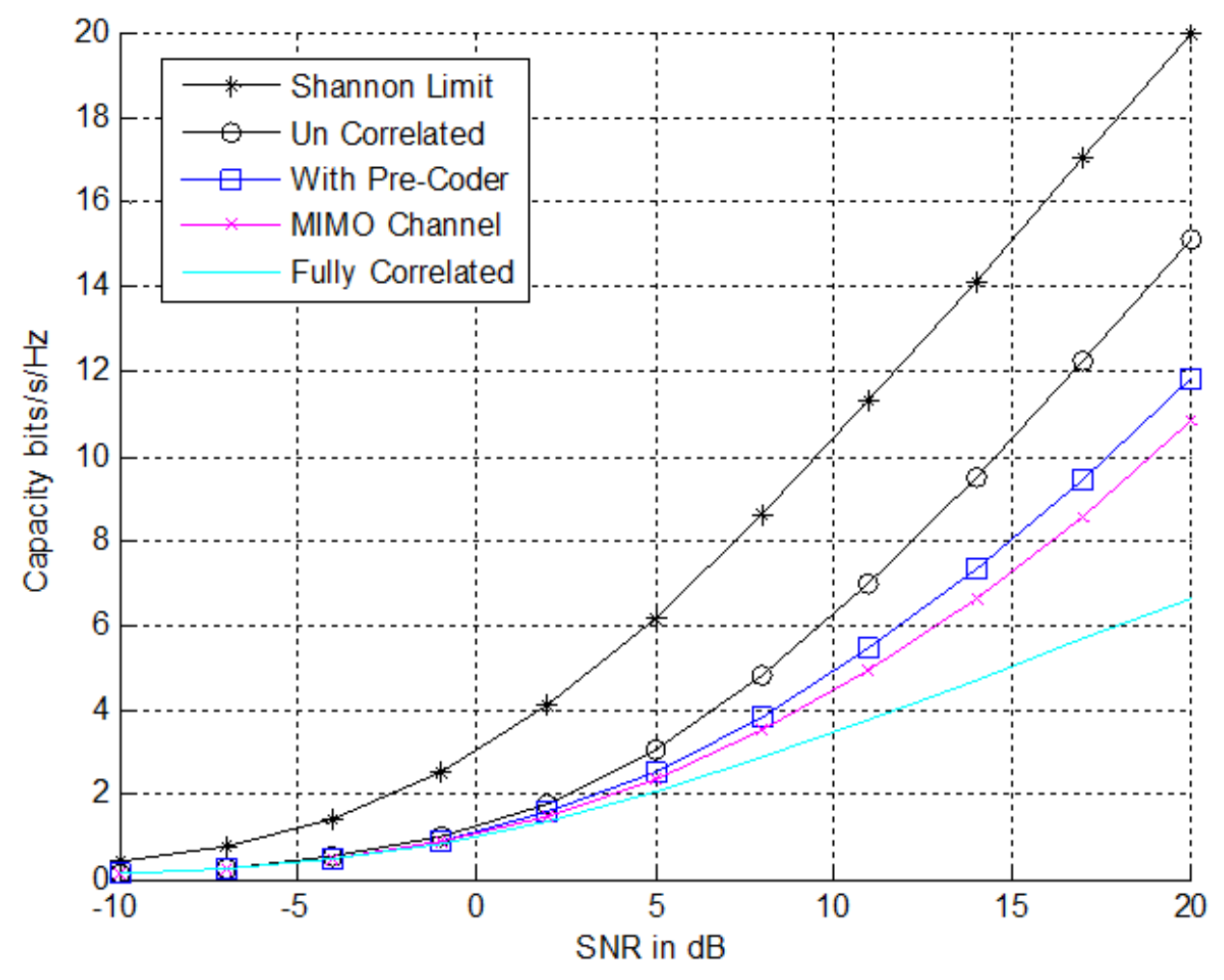

Fig. 5. MIMO capacity for three correlated rows 
The enhancement of row correlation was better than that of column correlation and the best achieved results were those of enhancing a system of a three correlated rows channel matrix. Fully correlated channels physically occurred in long distance scatter free wireless links which gives the lower capacity limit for a MIMO system.

The less capacity enhancement in the column correlation case is thought to be due to the high correlation of about $60-70 \%$ in other columns than the study ones. This problem was a cause of the high correlation between the random variables that MATLAB simulation generates and it is though that in real systems with less correlation between the other columns the proposed F's can even better enhance the capacity of correlated columns.

In this study a blind pre-coder was introduced and the results show that the blind pre-coder is a possible solution for column correlated channel matrix environment, whereas it does enhance the capacity for row correlated cases but with little amounts. Usually one can determine the kind of correlation in the system depending on the location of the $T_{x}$ and the $R_{x}$ and the existence of scatteres near. So if we are talking about a base station which lacks the existence of many scatteres this blind pre-coder is a good choice since it does enhance the capacity for column correlation in a good manner.

\section{Conclusion}

In this study, a MIMO system pre-coder is designed. The proposed pre-coder aims to maximize the capacity for MIMO system by attempting to de-correlate the channel matrix or in other words by trying to minimize the effect of high correlation in the channel matrix that degrades the MIMO system capacity and as a consequence full capacity gains will not be accomplished.

The goal of this design is to search for a suitable linear pre-coder with efficient and simple implementation and minimum complexity. The design depends only on the correlation of the channel matrix, as a consequence feedback channel from the receiver to the transmitter is not a requirement.

The presented blind pre-coder in this study is accomplished by conducting a searching algorithm using only $3 \times 3$ MIMO system. One of the open issues for future work is to examine the blind pre-coder with other configurations of MIMO systems.

\section{Author's Contributions}

Eman Alkhader: Participated in all experiments design and simulation, data acquisition and analysis and explanation of the final results.

Shereen Ismail: Contributed to summarizing the experimental data. Also she participated in proofreading of the final manuscript.

\section{Ethics}

This article is original and contains unpublished work. The corresponding author confirms that the other author has read and approved the manuscript and no ethical issues involved.

\section{References}

Al-Khader, E. and S. Ismail, 2015. Pre- and post-coding for capacity enhancement in MIMO. Proceedings of the 6th International Conference on Ambient Systems, Networks and Technologies, (SNT' 15), London, pp: 326-333.

Cho, Y.S., J. Kim, W. Yang and C. Kang, 2010. MIMOOFDM Wireless Communications with MATLAB. 1st Edn., John Wiley and Sons, Singapore, ISBN-10: 0470825626, pp: 544.

Foschini, G.J. and M.J. GANS, 1998. On limits of wireless communications in a fading environment when using multiple antennas. Wireless Personal Commun., 6: 311-335.

DOI: $10.1023 / \mathrm{A}: 1008889222784$

Hjørungnes, A., 2005. Precoding of orthogonal spacetime block codes in arbitrarily correlated MIMO channels: Iterative and closed-form solutions. IEEE Trans. Wireless Commun. DOI: 10.1109/TWC.2007.05271

Jung, M., K. Hwang and S. Choi, 2012. Multi-mode precoding scheme based on interference channel in MIMO-Based cognitive radio networks. Proceedings of the IEEE International Conference on ICT Convergence, Oct. 15-17, IEEE Xplore Press, pp: 227-232.

DOI: 10.1109/ICTC.2012.6386824

Noh, S., Y. Sung and M.D. Zoltowski, 2014. A new precoder design for blind channel estimation in MIMO-OFDM systems. IEEE Trans. Wireless Commun., 13: 7011-7024.

DOI: $10.1109 /$ TWC.2014.2354342

Rusek, F., D. Persson, B.K. Lau and E. Larsson, 2013. Scaling Up MIMO: Opportunities and challenges with very large arrays. IEEE Signal Process. Magaz., 30: 40-60.

DOI: 10.1109/MSP.2011.2178495

Shiu, D., G. Foschini, M. Gans and J. Kahn, 2000. Fading correlation and its effect on the capacity of multielement antenna systems. IEEE Trans. Commun., 48: 502-513. DOI: 10.1109/26.837052

Wang, C.X., F. Haider, X. Gao and X.H. You, 2014. Cellular architecture and key technologies for $5 \mathrm{G}$ wireless communication networks. IEEE Commun. Magaz., 52: 122-130.

DOI: 10.1109/MCOM.2014.6736752 
Eman Al Khader and Shereen S. Ismail / American Journal of Engineering and Applied Sciences 2017, 10 (1): 43.50 DOI: 10.3844/ajeassp.2017.43.50

Zaier, A. and R. Bouallègue, 2012. Blind channel estimation enhancement for MIMO-OFDM systems under high mobility conditions. Int. J. Wireless Mobile Netw., 4: 207-214.

DOI: $10.5121 /$ ijwmn.2012.4115
Zheng, K., L. Zhao, J. Mei, B. Shao and W. Xiang et al., 2015. Survey of large-scale MIMO systems. IEEE Commun. Surveys Tutorials, 17: 1738-1760. DOI: 10.1109/COMST.2015.2425294 